\title{
APPENDIX
}

\section{Major actors and staff for productions discussed in this volume}

\author{
Shakespeare Memorial Theatre, Stratford-upon-Avon, 1919 \\ Director: Nigel Playfair \\ Rosalind \\ Athene Seyler Silvius \\ Orlando \\ Geoffrey Kerr Phoebe \\ Clement Charles \\ Celia \\ Touchstone \\ Jaques \\ Oliver \\ Duke Senior \\ Marjory Holman Corin \\ Faith Faber \\ Nigel Playfair Duke Frederick Herbert Marshall \\ Herbert Marshall Adam \\ Gilbert Hare \\ Lionel Watts Amiens \\ Kinsey Peile Audrey \\ Bertram Binyon \\ Betty Chester
}

Théâtre de l'Atelier, Paris, 1934

Director: Jacques Copeau

$\begin{array}{lrlr}\text { Rosalind } & \text { Madeleine Lambert } & \text { Silvius } & \text { Lucien Arnaud } \\ \text { Orlando } & \text { Daniel Lecourtois } & \text { Phoebe } & \text { Madeleine Lauzun } \\ \text { Celia } & \text { Marie-Louise Delby } & \text { Corin } & \text { Guy Favières } \\ \text { Touchstone } & \text { Arthur Devère } & \text { Duke Frederick } & \text { Raymond Destac } \\ \text { Jaques } & \text { Jacques Copeau } & \text { Adam } & \text { Léonce Corne } \\ \text { Oliver } & \text { Jean Gournac } & \text { Amiens } & \text { Marcel d'Orval } \\ \text { Duke Senior } & \text { Geymond Vital } & \text { Audrey } & \text { Raymone Duchâteau }\end{array}$

Inter-Allied Film, London, 1936

Director: Paul Czinner

$\begin{array}{lrlr}\text { Rosalind } & \text { Elisabeth Bergner } & \text { Silvius } & \text { Richard Ainley } \\ \text { Orlando } & \text { Laurence Olivier } & \text { Phoebe } & \text { Joan White } \\ \text { Celia } & \text { Sophie Stewart } & \text { Corin } & \text { Aubrey Mather } \\ \text { Touchstone } & \text { Mackenzie Ward } & \text { Duke Frederick } & \text { Felix Aylme } \\ \text { Jaques } & \text { Leon Quartermaine } & \text { Adam } & \text { J. Fisher White } \\ \text { Oliver } & \text { John Laurie } & \text { Amiens } & \text { Stuart Robinson } \\ \text { Duke Senior } & \text { Henry Ainley } & \text { Audrey } & \text { Dorice Fordred }\end{array}$




\section{Royal Shakespeare Company, Stratford-upon-Avon and London, 1962-63}

Director: Michael Elliott

$\begin{array}{lrlr}\text { Rosalind } & \text { Vanessa Redgrave } & \text { Silvius } & \text { Peter McEnery } \\ \text { Orlando } & \text { Ian Bannen } & \text { Phoebe } & \text { Jill Dixon } \\ \text { Celia } & \text { Rosalind Knight } & \text { Corin } & \text { Russell Hunter } \\ \text { Touchstone } & \text { Colin Blakeley } & \text { Duke Frederick } & \text { Tony Church } \\ \text { Jaques } & \text { Max Adrian } & \text { Adam } & \text { Clifford Rose } \\ \text { Oliver } & \text { David Buck } & \text { Amiens } & \text { Eric Flynn } \\ \text { Duke Senior } & \text { Redmond Phillips } & \text { Audrey } & \text { Patsy Byrne }\end{array}$

\section{British Broadcasting Corporation, London, 1964}

Director: Ronald Eyre

$\begin{array}{lrlr}\text { Rosalind } & \text { Vanessa Redgrave } & \text { Silvius } & \text { Peter Gill } \\ \text { Orlando } & \text { Patrick Allen } & \text { Phoebe } & \text { Jeanne Hepple } \\ \text { Celia } & \text { Rosalind Knight } & \text { Corin } & \text { Russell Hunter } \\ \text { Touchstone } & \text { Patrick Wymark } & \text { Duke Frederick } & \text { Tony Church } \\ \text { Jaques } & \text { Max Adrian } & \text { Adam } & \text { Clifford Rose } \\ \text { Oliver } & \text { David Buck } & \text { Amiens } & \text { Eric Flynn } \\ \text { Duke Senior } & \text { Paul Hardwick } & \text { Audrey } & \text { Patsy Byrne }\end{array}$

\section{National Theatre, London, 1967-69}

Director: Clifford Williams

$\begin{array}{lrlr}\text { Rosalind } & \text { Ronald Pickup } & \text { Silvius } & \text { John McEnery } \\ \text { Orlando } & \text { Jeremy Brett } & \text { Phoebe } & \text { Richard Kay } \\ \text { Celia } & \text { Charles Kay } & \text { Corin } & \text { Gerald James } \\ \text { Touchstone } & \text { Derek Jacobi } & \text { Duke Frederick } & \text { Frank Wylie } \\ \text { Jaques } & \text { Robert Stephens } & \text { Adam } & \text { Harry Lomax } \\ \text { Oliver } & \text { Neil Fitzpatrick } & \text { Amiens } & \text { Roderick Horn } \\ \text { Duke Senior } & \text { Paul Curra } & \text { Audrey } & \text { Anthony Hopkins }\end{array}$

Royal Shakespeare Company, Stratford-upon-Avon, 1973

Director: Buzz Goodbody

$\begin{array}{lrlr}\text { Rosalind } & \text { Eileen Atkins } & \text { Silvius } & \text { Peter Machin } \\ \text { Orlando } & \text { David Suchet } & \text { Phoebe } & \text { Janet Cappell } \\ \text { Celia } & \text { Maureen Lipman } & \text { Corin } & \text { Jeffrey Dench } \\ \text { Touchstone } & \text { Derek Smith } & \text { Duke Frederick Clement McCallin } \\ \text { Jaques } & \text { Richard Pasco } & \text { Adam } & \text { Sydney Bromley } \\ \text { Oliver } & \text { Charles Keating } & \text { Amiens } & \text { Ray Armstrong } \\ \text { Duke Senior } & \text { Tony Church } & \text { Audrey } & \text { Annette Badland }\end{array}$




\section{Schaubühne, Berlin, 1977}

Director: Peter Stein

Rosalind

Orlando

Celia

Touchstone

Jaques

Oliver

Duke Senior
Jutta Lampe Silvius

Michael König Phoebe

Tina Engel Corin

Werner Rehm Duke Frederick

Peter Fitz Adam

Eberhard Feik Amiens

Günter Lampe Audrey
Wolf Redl

Elke Petri

Otto Mächtlinger

Otto Sander

Gerd David

Gerd Wameling

Libgart Schwartz

\section{British Broadcasting Corporation, London, 1978}

Director: Basil Coleman

Rosalind
Orlando
Celia
Touchstone
Jaques
Oliver
Duke Senior

$\begin{array}{rlr}\text { Helen Mirren } & \text { Silvius } & \text { Maynard Williams } \\ \text { Brian Stirner } & \text { Phoebe } & \text { Victoria Plucknett } \\ \text { Angharad Rees } & \text { Corin } & \text { David Lloyd Meredith } \\ \text { James Bolam } & \text { Duke Frederick } & \text { Richard Easton } \\ \text { Richard Pasco } & \text { Adam } & \text { Arthur Hewett } \\ \text { Clive Francis } & \text { Amiens } & \text { Tom McDonnell } \\ \text { Tony Church } & \text { Audrey } & \text { Marilyn Le Conte }\end{array}$

\section{Royal Shakespeare Company, Stratford-upon-Avon and London, 1985-86}

Director: Adrian Noble

$\begin{array}{lrlr}\text { Rosalind } & \text { Juliet Stevenson } & \text { Silvius } & \text { Roger Hyams } \\ \text { Orlando } & \text { Hilton McCrae } & \text { Phoebe } & \text { Lesley Manville } \\ \text { Celia } & \text { Fiona Shaw } & \text { Corin } & \text { Colin Douglas } \\ \text { Touchstone } & \text { Nicky Henson } & \text { Duke Frederick } & \text { Joseph O'Conor } \\ \text { Jaques } & \text { Alan Rickman } & \text { Adam } & \text { Mark Dignam } \\ \text { Oliver } & \text { Bruce Alexander } & \text { Amiens } & \text { Andrew Yeats } \\ \text { Duke Senior } & \text { Joseph O'Conor } & \text { Audrey } & \text { Mary Jo Randle }\end{array}$

\section{Cheek by Jowl, London and tour, 1991}

Director: Declan Donnellan

Rosalind
Orlando
Celia
Touchstone
Jaques
Oliver
Duke Senior

$\begin{aligned} \text { Adrian Lester } & \text { Silvius } \\ \text { Patrick Toomey } & \text { Phoebe } \\ \text { Tom Hollander } & \text { Corin } \\ \text { Peter Needham } & \text { Duke Frederick } \\ \text { Joe Dixon } & \text { Adam } \\ \text { Mark Bannister } & \text { Amiens } \\ \text { David Hobbs } & \text { Audrey }\end{aligned}$

Mark Benton

Sam Graham

Mike Afford

David Hobbs

Sam Graham

Conrad Nelson

Richard Cant 
Sands Films, London, 1992

Director: Christine Edzard

Rosalind

Emma Croft

Silvius

Ewen Bremmer

Orlando

Celia

Andrew Tiernan

Phoebe

Valerie Groga

Celia Bannerman

Corin

Roger Hammond

Touchstone

Griff Rhys Jones

Duke Frederick

Don Henderson

Jaques

James Fox

Adam

Andrew Tiernan Amiens

Cyril Cusack

Oliver

Don Henderson

Audrey

John Tams

Duke Senior

\section{Cheek by Jowl, London and tour, 1994-95}

Director: Declan Donnellan

\begin{tabular}{|c|c|c|c|}
\hline Rosalind & Adrian Lester & Silvius & Gavin Abbo \\
\hline Orlando & Scott Handy & Phoebe & Wayne Cat \\
\hline Celia & Simon Coates & Corin & Paul Kissau \\
\hline Touchstone & Peter Needham & Duke Frederick & David Ho \\
\hline Jaques & Michael Gardiner & Adam & Richard C \\
\hline Oliver & Jonathan Chesterman & Amiens & Rhashan Stc \\
\hline Duke Senior & David Hobbs & Audrey & Richard C \\
\hline
\end{tabular}

\section{BBC Films/HBO Films/Shakespeare Film Company, UK and USA, 2006}

Director: Kenneth Branagh

$\begin{array}{lrlr}\text { Rosalind } & \text { Bryce Dallas Howard } & \text { Silvius } & \text { Alex Wyndham } \\ \text { Orlando } & \text { David Oyelowo } & \text { Phoebe } & \text { Jade Jefferie } \\ \text { Celia } & \text { Romola Garai } & \text { Corin } & \text { Jimmy Yuill } \\ \text { Touchstone } & \text { Alfred Molina } & \text { Duke Frederick } & \text { Brian Blessed } \\ \text { Jaques } & \text { Kevin Kline } & \text { Adam } & \text { Richard Briers } \\ \text { Oliver } & \text { Adrian Lester } & \text { Amiens } & \text { Patrick Doyle } \\ \text { Duke Senior } & \text { Brian Blessed } & \text { Audrey } & \text { Janet McTeer }\end{array}$

Shakespeare's Globe, London, 2015

Director: Blanche McIntyre

$\begin{array}{lrlr}\text { Rosalind } & \text { Michelle Terry } & \text { Silvius } & \text { Jack Monagha } \\ \text { Orlando } & \text { Simon Harrison } & \text { Phoebe } & \text { Gwyneth Keyworth } \\ \text { Celia } & \text { Ellie Piercy } & \text { Corin } & \text { Patrick Driver } \\ \text { Touchstone } & \text { Daniel Crossley } & \text { Duke Frederick } & \text { David Beame } \\ \text { Jaques } & \text { James Garnon } & \text { Adam } & \text { Phil Whitchurch } \\ \text { Oliver } & \text { William Mannering } & \text { Amiens } & \text { William Mannering } \\ \text { Duke Senior } & \text { David Beames } & \text { Audrey } & \text { Sophia Nomvete }\end{array}$

